\title{
Differences in small noncoding RNAs profile between bull $X$ and Y sperm
}

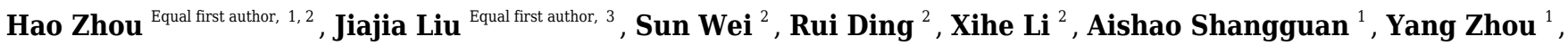 \\ Tesfaye Worku ${ }^{1}$, Xingjie Hao ${ }^{1}$, Faheem Ahmed Khan ${ }^{4}$, Liguo Yang ${ }^{1}$, Shujun Zhang ${ }^{\text {Corresp. } 1}$ \\ ${ }^{1}$ Key Laboratory of Agricultural Animal Genetics, Breeding and Reproduction, Education Ministry of China, College of Animal Science and Technology, \\ Huazhong Agricultural University, Wuhan, China \\ 2 Inner Mongolia Saikexing Institute of Breeding and Reproductive Biotechnology in Domestic Animal, Hohhot, China \\ 3 School of Biological Science and Technology, University of Jinan, Jinan, China \\ ${ }^{4}$ The Center for Biomedical Research, Tongji Hospital, Tongji Medical College, Huazhong University of Science and Technology, Wuhan, China \\ Corresponding Author: Shujun Zhang \\ Email address: sjxiaozhang@mail.hzau.edu.cn
}

The differences in small noncoding RNAs (sncRNAs), including miRNAs, piRNAs, and tRNAderived fragments (tsRNAs), between $X$ and $Y$ sperm of mammals remain unclear. Here, we employed high-throughput sequencing to systematically compare the sncRNA profiles of $X$ and $Y$ sperm from bulls $(n=3)$, which may have a wider implication for the whole mammalian class. For the comparison of miRNA profiles, we found that the abundance of bta-miR-652 and bta-miR-378 were significantly higher in X sperm, while nine miRNAs, including bta-miR-204 and bta-miR-3432a, had greater abundance in Y sperm $(p<0.05)$. qPCR was then used to further validate their abundances. Subsequent functional analysis revealed that their targeted genes in sperm were significantly involved in nucleosome binding and nucleosomal DNA binding. In contrast, their targeted genes in mature oocyte were significantly enriched in 11 catabolic processes, indicating that these differentially abundant miRNAs may trigger a series of catabolic processes for the catabolization of different $X$ and $Y$ sperm components during fertilization. Furthermore, we found that $X$ and $Y$ sperm showed differences in piRNA clusters distributed in the genome as well as piRNA and tsRNA abundance, two tsRNAs (tRNA-Ser-AGA and tRNA-Ser-TGA) had lower abundance in $X$ sperm than $Y$ sperm $(p<0.05)$. Overall, our work describes the different sncRNA profiles of $X$ and $Y$ sperm in cattle and enhances our understanding of their potential roles in the regulation of sex differences in sperm and early embryonic development . 


\section{Differences in small noncoding RNAs profile between} 3 bull $X$ and $Y$ sperm

4

5

6

7

8

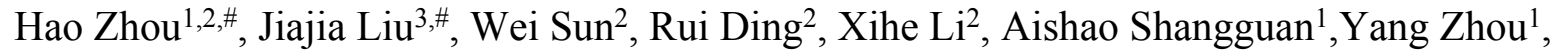
Tesfaye Worku ${ }^{1}$, Xingjie Hao ${ }^{1}$, Faheem Ahmed Khan ${ }^{4}$, Liguo Yang ${ }^{1}$, Shujun Zhang ${ }^{1, *}$

${ }^{1}$ Key Laboratory of Agricultural Animal Genetics, Breeding and Reproduction, Education Ministry of China, College of Animal Science and Technology, Huazhong Agricultural University, Wuhan, China

${ }^{2}$ Inner Mongolia Saikexing Institute of Breeding and Reproductive Biotechnology in Domestic Animal, Hohhot, China

${ }^{3}$ School of Biological Science and Technology, University of Jinan, Jinan, China

${ }^{4}$ The Center for Biomedical Research, Tongji Hospital, Tongji Medical College, Huazhong University of Science and Technology, Wuhan, China

\section{Corresponding Author:}

Shujun Zhang

No.1, Shizishan Street, Hongshan District, Wuhan, Hubei Province, 430070, China

Email address: sjxiaozhang@mail.hzau.edu.cn

\section{Abstract}

The differences in small noncoding RNAs (sncRNAs), including miRNAs, piRNAs, and tRNAderived fragments (tsRNAs), between $X$ and $Y$ sperm of mammals remain unclear. Here, we employed high-throughput sequencing to systematically compare the sncRNA profiles of $\mathrm{X}$ and $Y$ sperm from bulls $(n=3)$, which may have a wider implication for the whole mammalian class. For the comparison of miRNA profiles, we found that the abundance of bta-miR-652 and btamiR-378 were significantly higher in X sperm, while nine miRNAs, including bta-miR-204 and bta-miR-3432a, had greater abundance in Y sperm $(\mathrm{p}<0.05)$. qPCR was then used to further validate their abundances. Subsequent functional analysis revealed that their targeted genes in sperm were significantly involved in nucleosome binding and nucleosomal DNA binding. In contrast, their targeted genes in mature oocyte were significantly enriched in 11 catabolic processes, indicating that these differentially abundant miRNAs may trigger a series of catabolic processes for the catabolization of different $\mathrm{X}$ and $\mathrm{Y}$ sperm components during fertilization. Furthermore, we found that $\mathrm{X}$ and $\mathrm{Y}$ sperm showed differences in piRNA clusters distributed in the genome as well as piRNA and tsRNA abundance, two tsRNAs (tRNA-Ser-AGA and tRNASer-TGA) had lower abundance in X sperm than Y sperm $(p<0.05)$. Overall, our work describes the different sncRNA profiles of $\mathrm{X}$ and $\mathrm{Y}$ sperm in cattle and enhances our understanding of 
40 their potential roles in the regulation of sex differences in sperm and early embryonic

41 development.

42

43

\section{Introduction}

44 Several previous studies have considered the question of diversity between $\mathrm{X}$ and $\mathrm{Y}$ sperm, demonstrating significant variation in their structure, morphology, motility, and energy metabolism (Cui and Matthews, 1993; Sarkar et al., 1984; Shettles, 1960). With the advent of computer-assisted sperm analysis, which allows the objective evaluation of kinetic parameters, most of the detected variations have been considered controversial (Hossain et al., 2001; Penfold et al., 1998). Sperm carry different sex chromosomes (either an X- or a Y-chromosome), which provide clues for the discovery of other differences between $X$ and $Y$ sperm. Indeed, such differences have been identified in several different types of profiles (Yadav et al., 2017), including protein profiles (Chen et al., 2012; De Canio et al., 2014) and messenger RNA (mRNA) profiles (Chen et al., 2014), by using high-throughput measurement technologies. However, whether there is the difference in the profiles of sncRNAs is still unknown.

Recently, an increasing number of studies in several species have shown that mature ejaculate sperm carry thousands of sncRNA, including tRNA-derived fragments (tsRNAs), ribosomal RNAs (rRNAs) and small nucleolar RNAs (snoRNAs), especially microRNAs (miRNA) and Piwi-interacting RNAs (piRNA)(Sellem et al., 2020). As the best-studied type of small noncoding RNA, miRNA has been implicated in posttranscriptional control, by binding to an Argonaute (AGO) protein to stabilize its target through binding to the 3' untranslated region(UTR), and in the regulation of translation by targeting amino acid coding (CDS) regions (Hausser et al., 2013). Even though the complexity of sperm miRNA has been well characterized in several mammalian species, including humans (Pantano et al., 2015; Tay et al., 2008), mice (Nixon et al., 2015), pigs (Chen et al., 2017; Zhang et al., 2017) and bulls (Capra et al., 2017), the functions of most of the sperm miRNAs remain enigmatic. On one hand, sperm miRNAs seem to be required by the sperm themselves and may have a function that impacts sperm motility. As a snapshot of what remains after spermatogenesis, the sperm miRNA profile was shown to be altered in different types of motile sperm in bulls (Capra et al., 2017). On the other hand, sperm miRNAs may play roles in processes, such as fertilization (Yuan et al., 2016) and, subsequently embryonic development (Yuan et al., 2016), potentially even transmitting paternally acquired phenotypes (Grandjean et al., 2015; Rodgers et al., 2015; Sharma et al., 2015) after they are carried into the fertilized oocyte. The major reason why sperm miRNAs execute these roles is that in the fertilized oocyte they regulate target maternal mRNAs (Rodgers et al., 2015; Wang et al., 2017). miRNAs carried by sperm may control maternal mRNAs expression levels to affect epigenetic reprogramming, the cleavage, and apoptosis of somatic cell nuclear transfer (SCNT) embryosin cattle (Wang et al., 2017). Transgenerational effects associated with parental diet were also proposed to be mediated, at least in part, by sperm tsRNAs (Chen et al., 2016). In contrast to miRNAs ( 22nt), piRNAs (24-31 nucleotides ) are 
80

81

82

83

84

85

86

87

88

89

90

91

92

93

94

95

96

97

98

99

100

101

102

103

104

105

106

107

108

109

110

111

112

113

114

115

116

117

118

to the Ago-class (Aravin et al., 2006; Girard et al., 2006; Siomi et al., 2011). piRNAs are assumed to be produced from the polycistronic RNAs that are transcribed in the genome from a small number of specific regions named piRNA clusters. It has been proposed that piRNAs may protect genome integrity from the deleterious effects of repetitive and transposable elements by binding to the elements(Krawetz et al., 2011). piRNAs were shown to be the more abundant set of regulatory sncRNAs than other types of sncRNAs in human sperm (Pantano et al., 2015), their expression in sperm correlated to sperm concentration and fertilization rate(Cui et al., 2018). These groundbreaking detection of sperm sncRNA led us to question whether there are any differences in the sncRNA profiles of two types of sperm, and if so, what are the specific functions of sncRNA showing differences between $\mathrm{X}$ and $\mathrm{Y}$ sperm?

Here, we systematically compared the abundance of several kinds of sncRNA species between $\mathrm{X}$ and $\mathrm{Y}$ sperm, especially in miRNAs, piRNAs, and tsRNAs. To explore the roles that differentially abundant (DA) miRNAs play in sperm and fertilized oocyte, we predicted their target binding sites in 3'UTRs and CDS regions and performed Gene Ontology (GO) and Kyoto Encyclopedia of Genes and Genomes (KEGG) pathway enrichment analyses of the target genes presented in sperm and mature oocyte. To our knowledge, this study provides the first description of the differences in the sncRNA profiles of $\mathrm{X}$ and $\mathrm{Y}$ sperm, which could improve our understanding of their possible functions in the regulation of sex differences in sperm and early embryonic development.

\section{Materials \& Methods}

\section{Bull $X$ and $Y$ sperm collection}

Semen samples were obtained from the Saikexing Institute (Hohhot, China). Briefly, samples were collected from three Holstein bulls at three years of age. These bulls were fed the same diet daily and reared in the same conditions and environments. The semen was sampled from them using an artificial vagina and stored at room temperature $\left(18^{\circ} \mathrm{C}\right)$ for $1 \mathrm{~h}$. Subsequently, the samples were passed through a $50 \mu \mathrm{m}$ filter to remove debris or clumped sperm, and the sperm were stained with the Hoechst-33342 fluorophore (Sigma, St Louis, USA) via incubation at $34^{\circ} \mathrm{C}$ for $45 \mathrm{~min}$ in darkness. After staining, the three sperm samples were separated into three X sexsorted semen and three Y sex-sorted semen using a high-speed MoFlo SX XDP flow cytometer (DakoCytomation, Fort Collins, USA). The purity of X and Y sex-sorted semen were tested by using the sort reanalysis method (Welch and Johnson, 1999). In brief, $20 \mu 1$ semen from each sample sonicated to remove the sperm tail and stained with $20 \mu 1$ Hoechst-33342 fluorophore via incubation at $34^{\circ} \mathrm{C}$ for $20 \mathrm{~min}$ in darkness. These samples were input into the high-speed MoFlo SX XDP flow cytometer to measure the purity of X and Y sex-sorted semen by performing the resorting procedure. Then, the sorted semen was washed twice in phosphate buffered saline (PBS, GE Healthcare Life Sciences, USA) through centrifugation at $700 \mathrm{~g}$ for $10 \mathrm{~min}$ at $20^{\circ} \mathrm{C}$. We removed the supernatants and mixed the sperm pellets with TRIzol by incubation for $5 \mathrm{~min}$ at RT (Sigma; $0.5 \mathrm{ml}$ per $1 \times 10^{7} \mathrm{sperm}$ ). After that, the samples were store on dry ice for next 
119 day use. The Scientific Ethics Committee of Huazhong Agricultural University approved the 120 experimental design and animal treatments for the present study (permit number: HZAUSW121 2017-012), and all experimental protocols were conducted in accordance with the guidelines.

122 Sperm total RNA isolation, libraries preparation, and sequencing

123 The TRIzol method has been used to extract the sperm total RNA of the six samples (Das et al., 124 2010). Comprehensive protocols were outlined in our earlier report (Shangguan et al., 2020).

125 Using a 2100 Bioanalyzer, the RNA integrity was assessed after extraction of the RNA (Agilent 126 Technologies, USA). The validated RNAs of X and Y sperm (10 ng RNA from each sample) 127 were sequenced on the BGISEQ-500 platform (Fehlmann et al., 2016) at the BGI company 128 (Shenzhen, China). A sequencing library was prepared and sequenced according to a standard 129 protocol established by the BGI (Fehlmann et al., 2016).

\section{Preprocessing of small noncoding RNA data}

131

132

133

134

135

136

137

138

139

140

141

142

143

144

145

146

147

148

149

150

151

152

153

154

155

156

After sequencing, the raw data were obtained from $\mathrm{X}$ and $\mathrm{Y}$ sperm samples. The quality of sequencing reads was tested by fastQC (http://www.bioinformatics.babraham.ac.uk/projects/ fastqc/). The adapters were initially detached from the raw sequence data (3' adapters: AGTCGGAGGCCAAGCGGTCTTAGGAAGACAA, 5' adapters: GAACGACATGGCTACGATCCGACTT). Also, in order to obtain clean data, we used Trimmomatic to trim the low-quality bases of each sequence (Bolger et al., 2014). The options below were used to trim: SLIDINGWINDOW 4:15, MINLEN 15, MAXINFO 15:0.8. The sequencing data of X and Y sperm sncRNA have been deposited in the Sequence Read Archive (https://www.ncbi.nlm.nih.gov/sra) with the accession codes PRJNA624261.

\section{Small noncoding RNA annotation}

Analysis of the small noncoding RNA data was performed using Unitas with options: -tail 1 mismatch 0 (Gebert et al., 2017) (Unitas is a software for the classification and annotation of mature miRNAs, rRNAs, piRNAs, tsRNAs, protein-coding RNAs, small nucleolar RNAs (snoRNAs), small nuclear RNAs (snRNAs), low complexity RNAs, non-annotated RNAs, and miscellaneous RNAs (miscRNAs). To reduce false-positive results for miRNA annotation, Mirdeep2 (Friedländer et al., 2011) was also used to predict putative known mature miRNAs of Bos taurus. We chose 17-23 nt sequences (based on the most of the length of mature miRNA (Sendler et al., 2013) from the clean data from which the Bos taurus rRNA and tRNA sequences had been removed as the input for Mirdeep2. The known mature Bos taurus miRNAs dataset (miRBase v.21, http://www.miRbase.org/) was used for miRNA detection. Then, the relative abundance of all miRNAs annotated from two software was standardized to the transcripts per million reads value $(\mathrm{RPM})$ according to the formula: $\mathrm{RPM}=\left(\right.$ mapped reads $\left.\times 10^{6}\right) /$ total reads, and miRNAs with an RPM $>5$ that were found in at least 2 samples were identified as miRNAs expressed.

The information of piRNA cluster and tsRNAs of X and $\mathrm{Y}$ sperm was obtained from the output files of Unitas (version 2.1)(Rosenkranz and Zischler, 2012). We obtained piRNA clusters

Peer) reviewing PDF | (2020:06:49991:1:1:NEW 20 Jul 2020) 
157 data of each sample. For the piRNA cluster, if the genomic regions of two clusters identified in

158

159

160

161

162

163

164

165

166

167

168

169

170

171

172

173

174

175

176

177

178

179

180

181

182

183

184

185

186

187

188

189

190

191

192

193

194

195

one sample were overlapped on the genome, they were considered as the same cluster. The same clusters found in all 3 replications were identified as the conserved cluster in each group. Genes and repeats falling within the detected clusters were retrieved using bovinemine (http://bovinegenome.org). Furthermore, reads mapped within the detected clusters were also retrieved to map to all available piRNA database on piRBase (http://regulatoryrna.org/database/piRNA/download.html) to identify the putative piRNA using the Bowtie software(Langmead et al., 2009). piRNAs with an RPM > 10 that were annotated in at least three samples were defined as expressed piRNAs.

\section{Analysis of differential miRNA abundance}

Differentially abundant analysis was carried out using the Bioconductor DEseq2 $\mathrm{R}$ package (Love et al., 2014). By applying thresholds of a P-value $<0.05$ and $\mid \log 2$ (fold change) $\mid>1$, the remaining miRNAs were defined as significantly differentially abundant (DA) miRNAs.

Furthermore, the analysis of the differential abundance of piRNAs and tsRNAs between $\mathrm{X}$ and $\mathrm{Y}$ sperm was the same as that of miRNAs.

\section{Functional annotation of DA miRNAs}

Two datasets named transcriptome data sequenced from single bull metaphase II oocyte (GSE59186) $(\mathrm{n}=2)$ and Bull sperm transcriptome data(SRA055325) $(\mathrm{n}=1)$ that have been earlier published were applied to explore the function of $X$ and $Y$ sperm DA miRNAs. The raw sequencing data were collected from the Sequence Read Archive (SRA) and were reanalyzed following these processes: (1) We used Cutadapt (https://code.google.com/p/cutadapt/) to cut the sequencing adapters and FASTX-Toolkit (http://hannonlab.cshl.edu/fastx_toolkit/) was used to filter sequences of low quality with the options "fastq_quality_trimmer -v -Q 33 -1 30 -i -t 20"; (2) Clean reads were aligned to the reference genome (Btau_4.6.1) by Tophat software (Trapnell et al., 2012); (3) For each gene model the mapped sequencing was counted and recorded via Cufflinks in Fragments Per Kilobase Million (FPKM) (Trapnell et al., 2012). Moreover, sperm transcripts were filtered with FPKM $<50$, and oocyte genes with FPKM $>50$ were retained in at least one sample. Ultimately, we obtained 1,036 sperm genes and 2,584 oocyte genes (Table S5). miRwalk was applied to identify the targets of the DA miRNAs with TarPmiR-algorithms (http://129.206.7.150/) (Dweep and Gretz, 2015). miRNA binding sites including CDS and 3' UTR within the complete sequences of all Bos taurus genes were investigated. Only target genes with binding P-values $>0.8$ were retained for further analysis. The mature oocyte and sperm gene sets were further overlapped with the target genes set of DA miRNAs.

Functional annotations of the target genes found in sperm and matured oocyte were carried out by Clusterprofiler software, respectively ( $Y u$ et al., 2012). Genes acquired were subjected to enrichment analyses by GO and KEGG to detect the significantly enriched terms in target genes. Also, terms with an adjusted $\mathrm{p}<0.05$ by Benjamini-Hochberg $(\mathrm{BH})$ multiple testing were deemed significant.

Quantitative real-time PCR (qPCR) validation of the sequencing results 
196 To verify the accuracy of high throughput sequencing results, we randomly selected and 197 confirmed the abundance of four miRNAs (bta-miR-204, bta-miR-3432a, bta-miR-652, and bta198 miR-378) in X and Y sperms by qPCR. Sperm RNA was produced from another three bulls 199 semen following the aforementioned protocol. Using the miScript II RT Kit (Qiagen), total RNA 200 of each sample was reverse-transcribed into cDNA. qPCR was carried out on an ABI 7500 Real201 Time PCR system (Applied Biosystem) by using miScript SYBR Green PCR Kit (Qiagen) with a 202 miRNA-specific forward primer. The relative abundant levels of the miRNAs were normalized 203 to U6 and calculated by $2^{(-\Delta \Delta \mathrm{Ct})}$ approach. Table S11 shows the primer information.

204

205

206

207

208

209

210

211

212

213

214

\section{Read counts of each RNA class}

216

After sequencing, we obtained 33,974,607, 30,027,769 and 30,422,125 raw reads $(31,474,834 \pm$

217

$2,173,828$, mean $\pm \mathrm{SD}$ ) for X4069, X4118 and X4131 sample, respectively and 30,532,748,

218 29,907,341, and 29,594,201 raw reads $(30,011,430 \pm 477,853)$ for Y4069, Y4118 and Y4131

219 sample, respectively. The quality control results show that bases of these raw sequences are with

220 high quality score and the raw sequence lengths are $50 \mathrm{nt}$ in all samples, suggesting the good

221 quality of sequencing data we obtained (Additional material 1). After removing low-quality

222 reads, $25,037,734,26,837,611$, and 24,848,674 clean reads $(25,574,673 \pm 1,097,814)$ for X4069, X4118 and X4131 sample, respectively, and 28,164,513, 27,819,402, and 26,946,322 clean reads

224 $(27,643,412 \pm 627,875)$ for Y4069, Y4118 and Y4131 sample, respectively, remained. The

225 proportion of Bos taurus miRNA (11.9\% vs. $20.2 \%$, t-test, $\mathrm{p}=0.003786)$ and non-annotated

226 sequence $(68.1 \%$ vs. $52.7 \%$, t-test, $\mathrm{p}<0.0001)$ were significantly different between $\mathrm{X}$ and $\mathrm{Y}$

227 sperm, while the other small noncoding RNA species (miRNAs of other species, rRNAs,

228 tsRNAs, protein-coding RNAs, snoRNAs, miscRNAs, snRNAs, and piRNAs) presented similar

229 proportions (Table S1).

Identification of DA tsRNAs in $X$ and $Y$ sperm 
231 We identified 52 tsRNAs in $\mathrm{X}$ and $\mathrm{Y}$ sperm (Table S10), The comparison of $\mathrm{X}$ and $\mathrm{Y}$ sperm 232 revealed only 2 significantly DA tsRNAs, including tRNA-Ser-AGA and tRNA-Ser-TGA, both 233 they have lower abundance in the X sperm $(\mathrm{p}<0.05)$.

\section{Identification of DA miRNAs in $\mathrm{X}$ and $\mathrm{Y}$ sperm}

235 In total, 490 known Bos taurus miRNAs were detected by Unitas, and 202 known Bos taurus 236 miRNAs were identified by Mirdeep2, respectively. Among these miRNAs, 49 and 21 miRNAs

237

238

239

240

241

242

243

244

245

246

247

248

249

250

251

252

253

254

255

256

257

258

259

260

261

262

263

264

265

266

267

268

269 were differentially abundant at a significant level $(\mathrm{p}<0.05$ and $\mid \log 2$ fold change $\mid>1)$ (Table S2). We obtained 12 DA miRNAs that overlapped between 49 and 21 DA miRNAs identified by two different software, including 3 highly abundant miRNAs in X sperm (bta-miR-15a, bta-miR-652, and bta-miR-378) and 9 more enriched miRNAs in Y sperm (bta-miR-204, bta-miR-1271, btamiR-211, bta-miR-375, bta-miR-3432a, bta-miR-127, bta-miR-6529a, bta-miR-369-5p, and btamiR-196a) (Table 1). Among these DA miRNAs, bta-miR-204, bta-miR-375, and bta-miR-378 were the most significantly DA miRNAs $(\mathrm{p}<0.005)$. Bta-miR-204 $(\log 2 \mathrm{FC}=-2.36, \mathrm{P}=0.0002)$ was the most abundant miRNA in both fractions and was previously identified in human, pig, and mouse epididymal sperm (Table 2$)$. The abundance of bta-miR-652 $(\log 2 \mathrm{FC}=2.26, \mathrm{P}=$ $0.0092)$ that was greater in $X$ sperm was the only DA miRNA detected on the $X$ chromosome (Table 1), whose abundance has been detected in human and mouse epididymal sperm but not in boar sperm (Table 2)(Nixon et al., 2015; Pantano et al., 2015). Furthermore, the DA miRNAs (4/12) showed a higher preference for chromosome 21 (Table 1).

In addition to the $12 \mathrm{DA}$ miRNAs, another 118 Bos taurus miRNAs included the 10 most abundant non -DA miRNAs between $X$ and $Y$ sperm: bta-miR-100, bta-let-7a-5p, bta-miR-223p, bta-miR-151-5p, bta-miR-21-5p, bta-miR-99a-5p, bta-miR-16b, bta-miR-7, and bta-miR$27 \mathrm{a}-3 \mathrm{p}$ (Table S2). Interestingly, these miRNAs together accounted for $92 \%$ of the RPM values of all non-DA miRNAs, indicating that the levels of the miRNAs differed sharply. We also compared our findings with recent studies and found that bta-miR-100 is also present at high levels in porcine, bull, human, and mouse epididymal sperm miRNA profiles (Capra et al., 2017; Chen et al., 2017; Nixon et al., 2015; Pantano et al., 2015) (Table 2), suggesting that its biological functions are conserved across these species.

\section{Prediction of DA miRNA target genes in mature oocyte and sperm}

We predicted target sites in the 3'UTRs and CDS regions, of 1,677 and 4,028 genes, respectively, for nine upregulated DA miRNAs in Y sperm, and in 510 and 1224 genes, respectively, for three upregulated DA miRNAs in X sperm (Figure 1, Table S4). We found that a greater number of target genes were predicted to be bound in CDS regions than in 3'UTRs.

Similar to the prediction results, the number of predicted target genes present in mature oocyte and sperm that were bound at CDS sites was greater than the number bound at 3'UTR sites. In the mature oocyte gene sets (Table S5), 602 and 248 genes were targeted by nine upregulated DA miRNAs at CDS and 3'UTR binding sites, respectively, in Y sperm. Three upregulated DA miRNAs in X sperm targeted 163 and 70 genes in CDS and 3'UTR binding sites, respectively. In contrast, in the sperm gene sets (Table S 4), nine upregulated DA miRNAs 
270

271

272

273

274

275

276

277

278

279

280

281

282

283

284

285

286

287

288

289

290

291

292

293

294

295

296

297

298

299

300

301

302

303

304

305

306

307

308

of Y sperm targeted 146 and 37 genes at CDS and 3'UTR binding sites, respectively, and three upregulated DA miRNAs of X sperm targeted 33 and 11 genes at CDS and 3'UTR binding sites, respectively. (Figure 1, Table S6). Taken together, the results suggested that the phenomenon of miRNA regulation of gene expression through CDS regions may be widely present in sperm and fertilized oocyte. In addition, the DA miRNAs may exhibit one or more target genes, due to interacting with different target regions (Hausser et al., 2013). By removing these repeated target genes, we eventually obtained 887 target genes in the mature oocyte and 210 target genes in sperm (Figure 1). Among these target genes, 79\% (697/887) and 82\% (172/210) were bound at CDS regions in mature oocyte and sperm, respectively (Table S6). Furthermore, we found that $6.3 \%$ and $23.3 \%$ of oocyte genes were targeted by $\mathrm{X}$ and $\mathrm{Y}$ sperm upregulated DA miRNAs, respectively, on CDS sites, which was greater than $3.2 \%$ and $14.1 \%$ of sperm genes targeted by DA miRNAs on CDS sites (Figure 1). Similarly, on the 3'UTR sites, $2.7 \%$ and $9.6 \%$ of oocyte genes were targeted by $\mathrm{X}$ and $\mathrm{Y}$ sperm highly abundant miRNAs, respectively, which was higher than $1.1 \%$ and $3.6 \%$ of sperm genes targeted by DA miRNAs (Figure 1). These results suggested that DA miRNAs were more prone to target genes in matured oocyte than in sperm, which was consistent with the previous finding that miRNA targets are likely absent in sperm (Krawetz et al., 2011).

\section{Functional analysis of DA miRNA targets in sperm and mature oocyte}

In the functional enrichment analysis of the 210 targets in sperm, the top significantly enriched GO categories were mainly related to mRNA processing, nucleosome binding, and nucleosomal DNA binding (adjusted $\mathrm{p}<0.05$, Figure 2A, Table S7). Surprisingly, the 887 targets in mature oocyte were significantly related to 11 catabolic processes, including macromolecule catabolic processes, cellular protein catabolic processes, and organonitrogen compound catabolic processes (adjusted $\mathrm{p}<0.05$, Figure 2A, Table S7). The 17 genes (MAGOH, PSMC5, DICER1, KCTD13, TRIP12, EIF3E, PSMA5, UBE2H, PSMD11, USP1, SKP1, ARIH1, EZR, IDE, TIMP3, and TRIM13) related to catabolic processes were targeted by seven Y sperm upregulated DA miRNAs: bta-miR-127, bta-miR-1271, bta-miR-196a, bta-miR-204, bta-miR-3432a, bta-miR375 and bta-miR-6529a (Figure 2B, Table S 7). This finding may indicate that these DA miRNAs and their targets in the oocyte are involved in a series of catabolic processes. Here, no enriched GO terms overlapped between the sperm and mature oocyte (Figure 2A).

On the other hand, in the KEGG analysis of the 210 targets in sperm, seven KEGG pathways were significantly enriched, including the cell cycle and RNA transport (adjusted $\mathrm{p}<$ 0.05 , Table S7). In contrast, only the endometrial cancer pathway was significantly enriched by 887 targets in the mature oocyte (adjusted $\mathrm{p}<0.05$, Table S7).

\section{Different distributions of piRNA cluster between $X$ and $Y$ sperm}

Here, a total of 21 and 12 unique piRNA clusters loci were identified in X and Y sperm, respectively, and 71 clusters loci were shared between two fractions, cluster XY69 that was located in the region of $25,287,459 \mathrm{bp}$ to $25,335,935 \mathrm{bp}$ on chromosome 28 was reported to be conserved deeply in Eutherian mammals (Table S8). 
309

310

311

312

313

314

315

316

317

318

319

320

321

322

323

324

325

326

327

328

329

330

331

332

333

334

335

336

337

338

339

340

341

342

343

344

345

346

347

348

To best understand the potential functions of piRNAs in $\mathrm{X}$ and $\mathrm{Y}$ sperm, we searched genes and transposons falling within their unique cluster loci. 362 repeats and 14 genes were within the unique cluster region of X sperm. Of them, BovB, Bov-tA3, and Bov-A2 were the top three repeat elements detected in unique piRNA clusters of X sperm. Cluster X9 located on 14 chromosomes contained the greatest number of repeat elements (102) in all the clusters identified (Figure 3A, Table S9). 14 genes enriched in 14 GO terms (adjusted $\mathrm{p}<0.05$ ), including biology function of galactosyl ceramide catabolic process, and galactolipid metabolic process. On the other hand, 169 repeat elements and 7 genes are within unique cluster loci of $\mathrm{X}$ sperm, among them, 23 BovB was identified, which was the greatest number of repeat elements identified, followed by Bov-tA2 (21) and BOV-A2 (13). Cluster Y11 located on chromosome 24 contained the greatest number of 77 repeat elements (Figure 3A, Table S9). 13 GO terms were enriched by 7 genes (adjusted $\mathrm{p}<0.05$ ), including nucleosome assembly and histone H3-K27 trimethylation. Details of $\mathrm{X}$ and $\mathrm{Y}$ unique piRNA clusters including genes, repeats, and function of genes falling within the cluster regions are given in Figure3A and Table S9. Furthermore, the expressed piRNAs in $\mathrm{X}$ and $\mathrm{Y}$ sperm were explored, we identified 582 piRNAs. Of them, 28 piRNAs were differentially abundant $(\mathrm{p}<0.05), 15$ piRNAs had higher abundance in Y sperm and 13 piRNAs were enriched in X sperm. The most significantly enriched piRNAs in X and Y sperm are piR$5346348\left(\mathrm{p}=1.22 \times 10^{-12}\right)$ and piR-5342466 $\left(\mathrm{p}=1.13 \times 10^{-12}\right)$, respectively. (Figure $3 \mathrm{~B}$, Table S10).

\section{qPCR validation of DA miRNAs}

To validate the high-throughput sequencing results, we randomly selected four miRNAs (btamiR-204, bta-miR-3432a, bta-miR-652, and bta-miR-378) to perform the qPCR experiment. The relative fold changes of these miRNAs in qPCR were concordant with the sequencing results (Figure 4, Table S11), indicating that the miRNA identification and abundance estimation were reliable.

\section{Discussion}

In mammals, X sperm contains more DNA than Y sperm, and these DNA differences might result in differences in small RNA abundance. In our study, the differential abundance of miRNAs, piRNAs, and tsRNAs between the two types of sperm were identified. Previous studies have revealed that adjacent sperm cells can share gene products through intercellular bridges during spermatogenesis, suggesting that sncRNA molecules may be shared between $\mathrm{X}$ and $\mathrm{Y}$ sperm cell during spermatogenesis (Fawcett et al., 1959) and retained within mature sperm, which may explain why a part of non-DA small RNAs was identified between X and Y sperm. However, these products are probably not all shared through the intercellular bridge (Ventelä et al., 2003). Indeed, differences between $\mathrm{X}$ and $\mathrm{Y}$ sperm have been identified through several types of analyses, such as protein analysis (Chen et al., 2012; De Canio et al., 2014) and transcript analysis (Chen et al., 2014). As in previous studies (Chen et al., 2014; Chen et al., 
349

350

351

352

353

354

355

356

357

358

359

360

361

362

363

364

365

366

367

368

369

370

371

372

373

374

375

376

377

378

379

380

381

382

383

384

385

386

387

388

2012; De Canio et al., 2014), we used sex-sorted X and Y semen (including $~ 90 \% \mathrm{X}(\mathrm{Y})$ sperm and $\sim 10 \% \mathrm{Y}(\mathrm{X})$ sperm $)$ as the sequencing sample, which may also produce fewer DA miRNAs, due to the inevitable presence of false-negative DA miRNAs in the analytical results. Finally, by employing a conservative approach to identify DA miRNAs (only the DA miRNAs annotated from both Mirdeep2 and Unitas were included in the further analysis), the accuracy of DA miRNA identification was improved, but other DA miRNAs might have been missed.

In the present study, the total numbers of DA and highly abundant non-DA miRNAs were detected from previously reported data obtained from bull sperm (Capra et al., 2017; Sellem et al., 2020). Moreover, nine highly enriched non-DA miRNAs (bta-miR-100, let-7a-5p,bta-miR22-3p, bta-miR-151-5p, bta-miR-21-5p, bta-miR-99a-5p, bta-miR-16b, bta-miR-7 and bta-miR27a-3p) and two DA miRNAs (bta-miR-211, bta-miR-204, and bta-miR-375) were present among the top 20 abundant miRNAs of a previous study (Capra et al., 2017; Sellem et al., 2020) (Table 2). Some of the differences in sperm miRNA profiles observed in the current study may be due to the use of differences in the treatment of the samples. For example, in Capra et al.'s study, the sperm were cryopreserved in straws for AI(Capra et al., 2017), while sperm were stored on dry ice after sorting in the current study. Among the most abundant non-DA miRNAs, bta-miR-100 and bta-miR-151-5p have been shown to be associated with sperm motility in bulls (Capra et al., 2017). bta-miR-100, which exhibited the highest abundance in our study, has been previously reported as the most abundant in bull sperm (Stowe et al., 2014) and has been identified in sperm of other species: human, pig, and mouse (Table 2) (Chen et al., 2017; Nixon et al., 2015; Pantano et al., 2015). Additionally, bta-miR-100 was shown to exhibit low abundance in the semen of infertile males with semen abnormalities (Liu et al., 2012a), suggesting an important role of bta-miR-100 in regulating fertility across mammalian species.

Although sperm miRNA contents have been most extensively explored via high-throughput sequencing in mammals, the function of miRNAs in sperm itself essentially remains controversial. The main cause of this uncertainty is that mature sperm are widely thought to be translationally inactive in the cytoplasm (Park et al., 2012; Rahman et al., 2013). In a previously reported study of DA sperm proteins and mRNAs, we found that there were no DA proteins corresponding to mRNA which are differentially expressed between X and Y sperm (Chen et al., 2012; De Canio et al., 2014), which may provide indirect evidence of silencing of translation in sperm. Increasing evidence regarding miRNA-target interactions has revealed a new mode of miRNAs action through which gene translation may be regulated by miRNAs targeting CDS regions (Hausser et al., 2013; Tay et al., 2008). For instance, inhibition of translation in somatic cells was previously demonstrated to be due to miRNA binding sites located in CDS regions (Hausser et al., 2013). Similarly, miRNAs have been shown to cotarget the 3' UTRs and CDS regions of maternally expressed mRNAs to regulate embryonic development in early zebrafish embryos(Hausser et al., 2013). Furthermore, the application of high-throughput approaches for isolating argonaute-bound target sites indicates that CDS sites are as numerous as those located in 3' UTRs (Chi et al., 2009; Hafner et al., 2010). In our study, we found that most sperm mRNAs (82\%) and mature oocyte mRNAs (79\%) were predicted to be targeted by DE miRNAs 
389 through binding to CDS regions (Figure 1). In addition, the argonaute 2 complex, which is 390 crucial for miRNA function, was found to be bound to miRNAs in mouse sperm (Liu et al., 391 2012b). Here, it is likely that DA sperm miRNAs bind to CDS regions and act as translation392 inhibiting factors in sperm (Hosken and Hodgson, 2014), and mRNAs are regulated by the CDS 393 regions, which are widespread in sperm and fertilized oocyte. Furthermore, functional analysis of 394 DA miRNA-targeted genes in sperm showed that these genes were involved in nucleosome

396

397

398

399

400

401

402

403

404

405

406

407

408

409

410

411

412

413

414

415

416

417

418

419

420

421

422

423

424

425

426

427

428 binding and nucleosomal DNA binding. Mature sperm retains some fraction of residual nucleosomes(Balhorn et al., 1977). The X chromosome in X sperm was demonstrated to exhibit strong enrichment of nucleosome-binding sites, and the $\mathrm{Y}$ chromosome in $\mathrm{Y}$ sperm exhibited a strong depletion in bovine sperm (Samans et al., 2014). Overall, the results suggest that $\mathrm{X}$ and $\mathrm{Y}$ sperm, with different sex chromosomes, may contain genes targeted by DA miRNAs that perform different functions in nucleosome binding and nucleosomal DNA binding.

The egg is the ultimate destination for sperm, along with its miRNAs. Mammalian sperm carry subsets of miRNAs into oocyte during fertilization. However, whether sperm miRNAs can play the roles after fertilization is still controversial. One argument relevant to this issue is that the levels of sperm miRNA are low relative to those of unfertilized MII (Metaphase II) oocyte, and fertilization does not alter the MII oocyte miRNA repertoire, suggesting that it plays a limited role in mammalian fertilization or early preimplantation development (Amanai et al., 2006). However, an increasing number of studies have shown that sperm-borne miRNAs are indeed important for preimplantation embryonic development (Grandjean et al., 2015; Rodgers et al., 2015; Sharma et al., 2015). Shuiqiao Yuan et al found that sperm with altered miRNAs could fertilize wild-type eggs. However, embryos derived from these partial small noncoding RNA-deficient sperm displayed a significant reduction in developmental potential, which could be rescued by injecting wild-type sperm-derived total or small RNAs into ICSI (Intracytoplasmic sperm injection) embryos, whereas maternal miRNAs were found to be dispensable for both fertilization and preimplantation development(Yuan et al., 2016). According to recent studies, even when the content of sperm miRNAs is low, miRNAs can be involved in initiating a cascade of molecular events after fertilization, through targeted degradation of stored maternal mRNAs(Rodgers et al., 2015). In addition, the sperm-borne miR-449b can improve the first cleavage division, involve in epigenetic reprogramming and apoptotic status of preimplantation cloned bovine embryos through regulating maternal mRNAs (Wang et al., 2017). In the current study, DA miRNAs were more prone to target genes in matured oocyte than in sperm. Taken together, the results seem to indicate that one of the ways in which sperm miRNA perform their roles after fertilization is by regulating maternal genes. Based on this hypothesis, we carried out the functional analysis of the putative targets of DA miRNAs in the fertilized oocyte. The analysis of GO term annotations indicated that maternal mRNAs in oocyte targeted by DA miRNAs were significantly enriched in 11 catabolic processes. Seven DA miRNAs (bta-miR127, bta-miR-1271, bta-miR-196a, bta-miR-204, bta-miR-3432a, bta-miR-375 and bta-miR6529a), along with their 17 target genes were found to be involved in catabolic processes in the mature oocyte (Figure 3B). Among these miRNAs, miR-204 and miR-375 with their related

Peerj reviewing PDF | (2020:06:49991:1:1:NEW 20 Jul 2020) 
429 target genes have been well established to play a clear inhibitory role in catabolic processes in 430 cancer (Lin et al., 2017; Mao et al., 2016). Indeed, spermatozoon fertilization of oocyte was 431 previously demonstrated to trigger a selective process that recognizes and degrades paternally 432 inherited organelles (Al Rawi et al., 2011). Furthermore, X and Y sperm were reported to exhibit 433

434

435

436

437

438

439

440

441

442

443

444

445

446

447

448

449

450

451

452

453

454

455

456

457

458

459

460

461

462

463

464

465

466

467

468 different protein profiles (Chen et al., 2012; De Canio et al., 2014) and sex chromosome structures. Based on these enlightening findings, we postulated that, when an X sperm or Y sperm enters the oocyte, sperm-carried DA miRNAs probably have discriminating catabolic functions for $\mathrm{X}$ and $\mathrm{Y}$ sperm involving different components through regulating related genes. In the present study, thyroid hormone receptor interactor 12 (Trip12), a maternal gene that is putatively targeted by bta-miR-204, bta-miR-1271, bta-miR-375, and bta-miR-3432a, plays an important role in embryogenesis (Kajiro et al., 2011). Moreover, prolonged stress in mice was demonstrated to alter the expression of nine sperm miRNAs, including miR-204 and miR-375, which can regulate maternal mRNAs, resulting in changes in offspring hypothalamic-pituitaryadrenal (HPA) axis responses to stress (Rodgers et al., 2013; Rodgers et al., 2015). Interestingly, one of their targeted maternal mRNAs identified in mouse fertilized oocyte, Serine and arginine rich splicing factor 2 (Srsf2), was predicted to be targeted by another DA miRNAs bta-miR-378 in bull mature oocyte (Rodgers et al., 2013; Rodgers et al., 2015). HPA response patterns differ markedly in males and females (Handa et al., 1994; Kajantie and Phillips, 2006; Kudielka and Kirschbaum, 2005; Verma et al., 2011). These finding suggest that bta-miR-204, bta-miR-375 and bta-miR-378, three most significantly DE miRNAs, carried by X and Y sperm to the fertilized oocyte may regulate maternal mRNAs to potentially influence stress reactivity in the offspring. In addition to sperm miRNA, tsRNAs from sperm could act as acquired epigenetic factors and contribute to offspring phenotypes such as metabolic traits . In current study, two tsRNAs (tRNA-Ser-AGA and tRNA-Ser-TGA) were differentially abundant between X and Y sperm. The expression of tRNA-Ser-TGA was shown to be positively correlated with cell proliferation in prostate cancer cell, which could promote the transition of these cells from the gap 2 phase of the cell cycle to the mitotic phase (Lee et al., 2009). This finding suggest the possibility that tRNA-Ser-TGA may act in a similar manner upon delivery to the oocyte(Peng et al., 2012). However, the studies involved in the fields of embryo development related to these two DA tsRNAs remain limited, which still require further exploration.

PiRNAs are small noncoding RNAs that can have significant implications for germ cell development and function. piRNAs are shown to be the most abundant class of small RNAs in human sperm (Pantano et al. 2015). Sellem and colleagues found that $26 \%$ of reads were annotated by piRNAs in bull sperm (Sellem et al., 2020). In the present study, the percentage of clean reads mapping to piRNAs database was about $6.8 \%$ and $8.7 \%$ for $\mathrm{X}$ and $\mathrm{Y}$ sperm, respectively (Table S2). The differences in piRNA proportion observed between two studies may be mainly due to the use of different analysis strategies. The high modifications of a single nucleotide at either the $5 \mathrm{p}$ or $3 \mathrm{p}$ end were the most frequent changes (Sellem et al., 2020). In this study, the high threshold that allowed 0 mismatch when mapping the sequences to reference piRNA sequences was used for piRNAs annotation, which would increase the credibility of the 
469

470

471

472

473

474

475

476

477

478

479

480

481

482

483

484

485

486

\section{7}

488

489

490

491

492

493

494

495

496

497

498

499

500

501

502

503

504

505

506

507

508

509

510

511

conservative piRNA annotated results but decrease the proportion of piRNAs annotated. Genome mapping of such piRNA sequences revealed that piRNAs mostly originate from distinct genome clusters, termed piRNA clusters(Aravin et al., 2007), which are a few to hundreds of kb in length. The genomic locations of these loci are often conserved between related species such as mouse and human (Aravin et al., 2006; Gan et al., 2011), but the sequences of the piRNAs themselves have evolved rapidly differ even between closely related species such as human and chimpanzee (Lukic and Chen, 2011). In the present study, one piRNA cluster was reported to conserve deeply in eutherian mammals which are located between the CCAR1 and DDX50 genes were also identified in the present study and were named CXY69, it was conserved in X and Y sperm and also contain STOX1 gene. STOX1 transcript was antisense to the many piRNAs generated in CXY69 cluster (Chirn et al., 2015). The different distribution of piRNA clusters, containing different genes and transposons, and abundance of piRNAs between $\mathrm{X}$ and $\mathrm{Y}$ sperm were identified in this study, suggesting these piRNAs may play different regulatory roles between them. Because of the low level of piRNAs conservation between even closely related species (Girard et al., 2006; Hong et al., 2016; Krawetz et al., 2011; Lau et al., 2006), and studies deciphering the functions of piRNAs were still limited, the potential role of they played in $\mathrm{X}$ and $\mathrm{Y}$ sperm remain to be further understood.

\section{Conclusions}

In conclusion, the present study revealed the sncRNA contents of X and Y sperm and highlighted the differences in the abundance and diversity of several common sncRNAs across two types of sperm. Additionally, we comprehensively discussed the roles of the DA miRNAs in sperm and fertilized oocyte, which could enhance our understanding of their potential functions involved in sex differences in sperm and early embryonic development.

\section{Acknowledgments}

We thank Dr. Denis Larkin and Dr. Marta Farré for valuable comments on the manuscript.

\section{References}

Al Rawi S, Louvet-Vallée S, Djeddi A, Sachse M, Culetto E, Hajjar C, Boyd L, Legouis R, and Galy V. 2011. Postfertilization autophagy of sperm organelles prevents paternal mitochondrial DNA transmission. Science 334:1144-1147.

Amanai M, Brahmajosyula M, and Perry AC. 2006. A restricted role for sperm-borne microRNAs in mammalian fertilization. Biol Reprod 75:877-884. 10.1095/biolreprod.106.056499

Aravin A, Gaidatzis D, Pfeffer S, Lagos-Quintana M, Landgraf P, lovino N, Morris P, Brownstein MJ, Kuramochi-Miyagawa S, Nakano T, Chien M, Russo JJ, Ju J, Sheridan R, Sander C, Zavolan M, and Tuschl T. 2006. A novel class of small RNAs bind to MILI protein in mouse testes. Nature 442:203-207. 10.1038/nature04916

Aravin AA, Sachidanandam R, Girard A, Fejes-Toth K, and Hannon GJ. 2007. Developmentally regulated piRNA clusters implicate MILI in transposon control. Science 316:744-747. 10.1126/science.1142612

Balhorn R, Gledhill BL, and Wyrobek AJ. 1977. Mouse sperm chromatin proteins: quantitative isolation and partial characterization. Biochemistry 16:4074-4080. 10.1021/bi00637a021 
512

513

514

515

516

517

518

519

520

521

522

523

524

525

526

527

528

529

530

531

532

533

534

535

536

537

538

539

540

541

542

543

544

545

546

547

548

549

550

551

552

553

554

555

556

557

558

559

560
Bolger AM, Lohse M, and Usadel B. 2014. Trimmomatic: a flexible trimmer for Illumina sequence data. Bioinformatics 30:2114-2120.

Capra E, Turri F, Lazzari B, Cremonesi P, Gliozzi TM, Fojadelli I, Stella A, and Pizzi F. 2017. Small RNA sequencing of cryopreserved semen from single bull revealed altered miRNAs and piRNAs expression between High- and Low-motile sperm populations. BMC Genomics 18:14. 10.1186/s12864-016-3394-7

Card CJ, Anderson EJ, Zamberlan S, Krieger KE, Kaproth M, and Sartini BL. 2013. Cryopreserved Bovine Spermatozoal Transcript Profile as Revealed by High-Throughput Ribonucleic Acid Sequencing1. Biology of Reproduction 88:49, 41-49-49, 41-49. 10.1095/biolreprod.112.103788

Chen C, Wu H, Shen D, Wang S, Zhang L, Wang X, Gao B, Wu T, Li B, and Li K. 2017. Comparative profiling of small RNAs of pig seminal plasma and ejaculated and epididymal sperm. Reproduction 153:785-796.

Chen Q, Yan M, Cao Z, Li X, Zhang Y, Shi J, Feng GH, Peng H, Zhang X, Zhang Y, Qian J, Duan E, Zhai Q, and Zhou Q. 2016. Sperm tsRNAs contribute to intergenerational inheritance of an acquired metabolic disorder. Science 351:397-400. 10.1126/science.aad7977

Chen X, Yue Y, He Y, Zhu H, Hao H, Zhao X, Qin T, and Wang D. 2014. Identification and characterization of genes differentially expressed in $X$ and $Y$ sperm using suppression subtractive hybridization and cDNA microarray. Molecular reproduction and development 81:908-917.

Chen X, Zhu H, Wu C, Han W, Hao H, Zhao X, Du W, Qin T, Liu Y, and Wang D. 2012. Identification of differentially expressed proteins between bull $X$ and $Y$ spermatozoa. Journal of proteomics 77:59-67.

Chi SW, Zang JB, Mele A, and Darnell RB. 2009. Argonaute HITS-CLIP decodes microRNAmRNA interaction maps. Nature 460:479.

Chirn G-w, Rahman R, Sytnikova YA, Matts JA, Zeng M, Gerlach D, Yu M, Berger B, Naramura M, Kile BT, and Lau NC. 2015. Conserved piRNA Expression from a Distinct Set of piRNA Cluster Loci in Eutherian Mammals. PLoS Genetics 11:e1005652. 10.1371/journal.pgen.1005652

Cui KH, and Matthews CD. 1993. X larger than Y. Nature 366:117-118. 10.1038/366117b0

Cui L, Fang L, Shi B, Qiu S, and Ye Y. 2018. Spermatozoa Expression of piR-31704, piR39888, and piR-40349 and Their Correlation to Sperm Concentration and Fertilization Rate After ICSI. Reproductive Sciences 25:733-739. 10.1177/1933719117725822

Das PJ, Paria N, Gustafson-Seabury A, Vishnoi M, Chaki SP, Love CC, Varner DD, Chowdhary BP, and Raudsepp T. 2010. Total RNA isolation from stallion sperm and testis biopsies. Theriogenology 74:1099-1106. e1092.

De Canio M, Soggiu A, Piras C, Bonizzi L, Galli A, Urbani A, and Roncada P. 2014. Differential protein profile in sexed bovine semen: shotgun proteomics investigation. Molecular BioSystems 10:1264-1271.

Dweep H, and Gretz N. 2015. miRWalk2. 0: a comprehensive atlas of microRNA-target interactions. Nature methods 12:697.

Fawcett DW, Ito S, and Slautterback D. 1959. The occurrence of intercellular bridges in groups of cells exhibiting synchronous differentiation. The Journal of Cell Biology 5:453-460.

Fehlmann T, Reinheimer S, Geng C, Su X, Drmanac S, Alexeev A, Zhang C, Backes C, Ludwig N, Hart M, An D, Zhu Z, Xu C, Chen A, Ni M, Liu J, Li Y, Poulter M, Li Y, Stähler C, Drmanac R, Xu X, Meese E, and Keller A. 2016. cPAS-based sequencing on the BGISEQ-500 to explore small non-coding RNAs. Clin Epigenetics 8:123. 10.1186/s13148-016-0287-1 
561

562

563

564

565

566

567

568

569

570

571

572

573

574

575

576

577

578

579

580

581

582

583

584

585

586

587

588

589

590

591

592

593

594

595

596

597

598

599

600

601

602

603

604

605

606

607

608

609

610

611

Friedländer MR, Mackowiak SD, Li N, Chen W, and Rajewsky N. 2011. miRDeep2 accurately identifies known and hundreds of novel microRNA genes in seven animal clades. Nucleic acids research 40:37-52.

Gan H, Lin X, Zhang Z, Zhang W, Liao S, Wang L, and Han C. 2011. piRNA profiling during specific stages of mouse spermatogenesis. RNA (New York, NY) 17:1191-1203. 10.1261/rna.2648411

Gebert D, Hewel C, and Rosenkranz D. 2017. unitas: the universal tool for annotation of small RNAs. BMC Genomics 18:644.

Girard A, Sachidanandam R, Hannon GJ, and Carmell MA. 2006. A germline-specific class of small RNAs binds mammalian Piwi proteins. Nature 442:199.

Grandjean V, Fourré S, De Abreu DAF, Derieppe M-A, Remy J-J, and Rassoulzadegan M. 2015. RNA-mediated paternal heredity of diet-induced obesity and metabolic disorders. Scientific reports 5:18193.

Hafner M, Landthaler M, Burger L, Khorshid M, Hausser J, Berninger P, Rothballer A, Ascano Jr M, Jungkamp A-C, and Munschauer M. 2010. Transcriptome-wide identification of RNAbinding protein and microRNA target sites by PAR-CLIP. Cell 141:129-141.

Handa RJ, Burgess LH, Kerr JE, and O'Keefe JA. 1994. Gonadal steroid hormone receptors and sex differences in the hypothalamo-pituitary-adrenal axis. Hormones and behavior 28:464-476.

Hausser J, Syed AP, Bilen B, and Zavolan M. 2013. Analysis of CDS-located miRNA target sites suggests that they can effectively inhibit translation. Genome research 23:604-615.

Hong Y, Wang C, Fu Z, Liang H, Zhang S, Lu M, Sun W, Ye C, Zhang C-Y, and Zen K. 2016. Systematic characterization of seminal plasma piRNAs as molecular biomarkers for male infertility. Scientific reports 6.

Hosken DJ, and Hodgson DJ. 2014. Why do sperm carry RNA? Relatedness, conflict, and control. Trends in ecology \& evolution 29:451-455.

Hossain AM, Barik S, and Kulkarni PM. 2001. Lack of significant morphological differences between human $X$ and $Y$ spermatozoa and their precursor cells (spermatids) exposed to different prehybridization treatments. Journal of andrology 22:119-123.

Jiang Z, Sun J, Dong H, Luo O, Zheng X, Obergfell C, Tang Y, Bi J, O'Neill R, and Ruan Y. 2014. Transcriptional profiles of bovine in vivo pre-implantation development. BMC Genomics 15:756.

Johnson G, Sendler E, Lalancette C, Hauser R, Diamond MP, and Krawetz S. 2011. Cleavage of rRNA ensures translational cessation in sperm at fertilization. Molecular human reproduction 17:721-726.

Kajantie E, and Phillips DI. 2006. The effects of sex and hormonal status on the physiological response to acute psychosocial stress. Psychoneuroendocrinology 31:151-178. 10.1016/j.psyneuen.2005.07.002

Kajiro M, Tsuchiya M, Kawabe Y-i, Furumai R, Iwasaki N, Hayashi Y, Katano M, Nakajima Y, Goto N, and Watanabe T. 2011. The E3 ubiquitin ligase activity of Trip12 is essential for mouse embryogenesis. PLoS One 6:e25871.

Krawetz SA, Kruger A, Lalancette C, Tagett R, Anton E, Draghici S, and Diamond MP. 2011. A survey of small RNAs in human sperm. Human Reproduction 26:3401-3412. 10.1093/humrep/der329

Kudielka BM, and Kirschbaum C. 2005. Sex differences in HPA axis responses to stress: a review. Biol Psychol 69:113-132. 10.1016/j.biopsycho.2004.11.009

Langmead B, Trapnell C, Pop M, and Salzberg SL. 2009. Ultrafast and memory-efficient alignment of short DNA sequences to the human genome. Genome Biology 10:R25. 10.1186/gb-2009-10-3-r25

Lau NC, Seto AG, Kim J, Kuramochi-Miyagawa S, Nakano T, Bartel DP, and Kingston RE. 2006. Characterization of the piRNA complex from rat testes. Science 313:363-367.

Peer) reviewing PDF | (2020:06:49991:1:1:NEW 20 Jul 2020) 
612

613

614

615

616

617

618

619

620

621

622

623

624

625

626

627

628

629

630

631

632

633

634

635

636

637

638

639

640

641

642

643

644

645

646

647

648

649

650

651

652

653

654

655

656

657

658

659

660

661

662

Lee YS, Shibata Y, Malhotra A, and Dutta A. 2009. A novel class of small RNAs: tRNA-derived RNA fragments (tRFs). Genes Dev 23:2639-2649. 10.1101/gad.1837609

Lin Y-C, Lin J-F, Tsai T-F, Chou K-Y, Chen H-E, Thomas I, and Hwang S. 2017. Tumor suppressor miRNA-204-5p promotes apoptosis by targeting BCL2 in prostate cancer cells. Asian journal of surgery 40:396-406.

Liu T, Cheng W, Gao Y, Wang H, and Liu Z. 2012a. Microarray analysis of microRNA expression patterns in the semen of infertile men with semen abnormalities. Molecular medicine reports 6:535-542.

Liu W-M, Pang RT, Chiu PC, Wong BP, Lao K, Lee K-F, and Yeung WS. 2012b. Sperm-borne microRNA-34c is required for the first cleavage division in mouse. Proceedings of the National Academy of Sciences 109:490-494.

Love MI, Huber W, and Anders S. 2014. Moderated estimation of fold change and dispersion for RNA-seq data with DESeq2. Genome Biology 15:550.

Lukic S, and Chen K. 2011. Human piRNAs Are Under Selection in Africans and Repress Transposable Elements. Molecular Biology \& Evolution 28:3061.

Mao Q, Quan T, Luo B, Guo X, Liu L, and Zheng Q. 2016. MiR-375 targets KLF4 and impacts the proliferation of colorectal carcinoma. Tumor Biology 37:463-471.

Mao S, Sendler E, Goodrich RJ, Hauser R, and Krawetz SA. 2014. A comparison of sperm RNA-seq methods. Systems biology in reproductive medicine 60:308-315.

Nixon B, Stanger SJ, Mihalas BP, Reilly JN, Anderson AL, Tyagi S, Holt JE, and McLaughlin EA. 2015. The MicroRNA Signature of Mouse Spermatozoa Is Substantially Modified During Epididymal Maturation. Biology of Reproduction 93. 10.1095/biolreprod.115.132209

Pantano L, Jodar M, Bak M, Ballescà JL, Tommerup N, Oliva R, and Vavouri T. 2015. The small RNA content of human sperm reveals pseudogene-derived piRNAs complementary to protein-coding genes. Rna 21:1085-1095.

Park Y-J, Kwon W-S, Oh S-A, and Pang M-G. 2012. Fertility-related proteomic profiling bull spermatozoa separated by percoll. Journal of proteome research 11:4162-4168.

Penfold L, Holt C, Holt W, Welch G, Cran D, and Johnson L. 1998. Main content area Comparative motility of $X$ and $Y$ chromosome-bearing bovine sperm separated on the basis of DNA content by flow sorting. Molecular reproduction and development 50:323327.

Peng H, Shi J, Zhang Y, Zhang H, Liao S, Li W, Lei L, Han C, Ning L, Cao Y, Zhou Q, Chen Q, and Duan E. 2012. A novel class of tRNA-derived small RNAs extremely enriched in mature mouse sperm. Cell Res 22:1609-1612. 10.1038/cr.2012.141

Rahman MS, Lee J-S, Kwon W-S, and Pang M-G. 2013. Sperm proteomics: road to male fertility and contraception. International journal of endocrinology 2013.

Rodgers AB, Morgan CP, Bronson SL, Revello S, and Bale TL. 2013. Paternal stress exposure alters sperm microRNA content and reprograms offspring HPA stress axis regulation. Journal of Neuroscience 33:9003-9012.

Rodgers AB, Morgan CP, Leu NA, and Bale TL. 2015. Transgenerational epigenetic programming via sperm microRNA recapitulates effects of paternal stress. Proceedings of the National Academy of Sciences 112:13699-13704.

Rosenkranz D, and Zischler H. 2012. proTRAC-a software for probabilistic piRNA cluster detection, visualization and analysis. BMC bioinformatics 13:5.

Samans B, Yang Y, Krebs S, Sarode Gaurav V, Blum H, Reichenbach M, Wolf E, Steger K, Dansranjavin T, and Schagdarsurengin U. 2014. Uniformity of Nucleosome Preservation Pattern in Mammalian Sperm and Its Connection to Repetitive DNA Elements. Developmental Cell 30:23-35. https://doi.org/10.1016/j.devcel.2014.05.023

Sarkar S, Jolly DJ, Friedmann T, and Jones OW. 1984. Swimming behavior of $X$ and $Y$ human sperm. Differentiation 27:120-125.

Peer) reviewing PDF | (2020:06:49991:1:1:NEW 20 Jul 2020) 
663

664

665

666

667

668

669

670

671

672

673

674

675

676

677

678

679

680

681

682

683

684

685

686

687

688

689

690

691

692

693

694

695

696

697

698

699

700

701

702

703

704

705

706

707

708

709

Sellem E, Marthey S, Rau A, Jouneau L, Bonnet A, Perrier J-P, Fritz S, Le Danvic C, Boussaha $\mathrm{M}$, Kiefer H, Jammes H, and Schibler L. 2020. A comprehensive overview of bull spermborne small non-coding RNAs and their diversity across breeds. Epigenetics \& chromatin 13:19. 10.1186/s13072-020-00340-0

Sendler E, Johnson GD, Mao S, Goodrich RJ, Diamond MP, Hauser R, and Krawetz SA. 2013. Stability, delivery and functions of human sperm RNAs at fertilization. Nucleic acids research 41:4104-4117.

Shangguan A, Zhou H, Sun W, Ding R, Li X, Liu J, Zhou Y, Chen X, Ding F, Yang L, and Zhang S. 2020. Cryopreservation Induces Alterations of miRNA and mRNA Fragment Profiles of Bull Sperm. Front Genet 11. 10.3389/fgene.2020.00419

Sharma U, Conine CC, Shea JM, Boskovic A, Derr AG, Bing XY, Belleannee C, Kucukural A, Serra RW, and Sun F. 2015. Biogenesis and function of tRNA fragments during sperm maturation and fertilization in mammals. Science:aad6780.

Shettles LB. 1960. Nuclear morphology of human spermatozoa. Nature 186:648-649.

Siomi MC, Sato K, Pezic D, and Aravin AA. 2011. PIWI-interacting small RNAs: the vanguard of genome defence. Nature Reviews Molecular Cell Biology 12:246.

Stowe HM, Calcatera SM, Dimmick MA, Andrae JG, Duckett SK, and Pratt SL. 2014. The bull sperm microRNAome and the effect of fescue toxicosis on sperm microRNA expression. PLoS One 9:e113163.

Tay Y, Zhang JQ, Thomson AM, Lim B, and Rigoutsos I. 2008. MicroRNAs to Nanog, Oct4 and Sox2 coding regions modulate embryonic stem cell differentiation. Nature 455:1124U1112. 10.1038/nature07299

Ventelä S, Toppari J, and Parvinen M. 2003. Intercellular organelle traffic through cytoplasmic bridges in early spermatids of the rat: mechanisms of haploid gene product sharing. Molecular biology of the cell 14:2768-2780.

Verma R, Balhara YPS, and Gupta CS. 2011. Gender differences in stress response: Role of developmental and biological determinants. Industrial psychiatry journal 20:4.

Wang M, Gao Y, Qu P, Qing S, Qiao F, Zhang Y, Mager J, and Wang Y. 2017. Sperm-borne miR-449b influences cleavage, epigenetic reprogramming and apoptosis of SCNT embryos in bovine. Scientific reports 7:13403. 10.1038/s41598-017-13899-8

Welch GR, and Johnson LA. 1999. Sex preselection: Laboratory validation of the sperm sex ratio of flow sorted X-and Y-sperm by sort reanalysis for DNA. Theriogenology 52:13431352. https://doi.org/10.1016/S0093-691X(99)00221-6

Yadav SK, Gangwar DK, Singh J, Tikadar CK, Khanna VV, Saini S, Dholpuria S, Palta P, Manik RS, and Singh MK. 2017. An immunological approach of sperm sexing and different methods for identification of X-and Y-chromosome bearing sperm. Veterinary World 10:498.

Yu G, Wang L-G, Han Y, and He Q-Y. 2012. clusterProfiler: an R package for comparing biological themes among gene clusters. Omics: a journal of integrative biology 16:284287.

Yuan S, Schuster A, Tang C, Yu T, Ortogero N, Bao J, Zheng H, and Yan W. 2016. Spermborne miRNAs and endo-siRNAs are important for fertilization and preimplantation embryonic development. Development (Cambridge, England) 143:635-647. 10.1242/dev.131755

Zhang Y, Dai D, Chang Y, Li Y, Zhang M, Zhou G, Peng Z, and Zeng C. 2017. Cryopreservation of boar sperm induces differential microRNAs expression. Cryobiology 76:24-33.

Peer) reviewing PDF | (2020:06:49991:1:1:NEW 20 Jul 2020) 


\section{Figure 1}

Upset plot of intersections between gene set targeted by differentially abundant miRNA through the 3'UTR region and CDS region and sperm or matured oocyte gene set.

The bar chart on the left indicates target (dark gray) sperm (black) and matured oocyte (light gray) gene sets. The upper bar chart indicates the intersection size. Black bar refers to target genes in sperm, light gray bar refers to target genes in matured oocyte. The number over the bar indicates the number of genes. The percentage in brackets refers to the proportion of the target genes in the sperm genes or matured oocyte genes. Dark connected dots on the bottom panel indicate which substrates are considered for each intersection.

X/Y_UP_3UTR/CDS refer to genes targeted by up-regulated DA miRNAs in X/Y sperm though binding 3UTR/CDS region. 

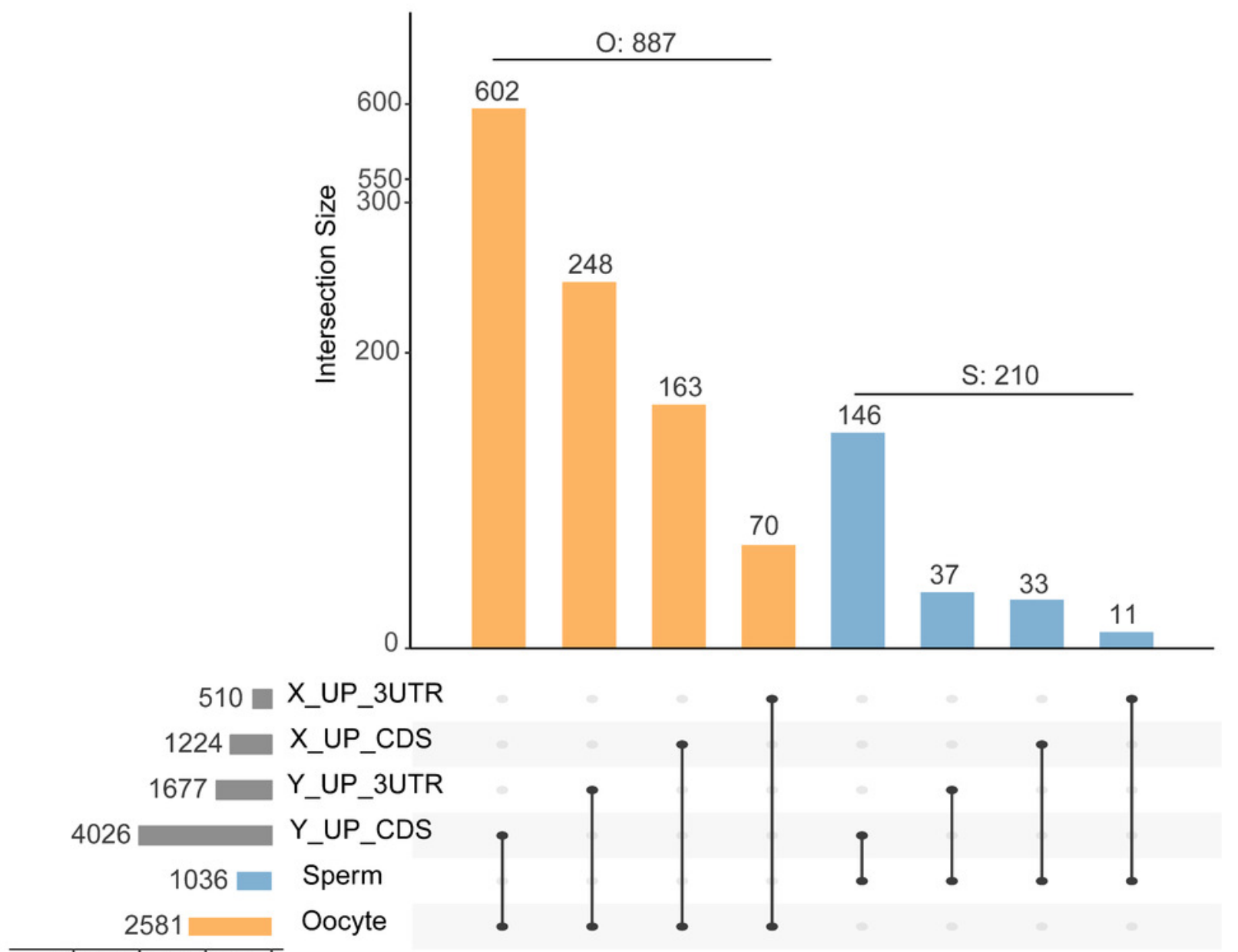

6000400020000

Set Size 


\section{Figure 2}

Functional analysis of differentially abundant miRNAs with their predicted target genes presented in sperm and mature oocyte.

(A) Bar plot shows the significant (adjusted P-value < 0.05) gene ontology (GO) terms enriched by the target genes in sperm and mature oocyte. Bar height shows the enrichment scores (-log adjusted P-value) of the GO terms. Line plot depicts the number of genes that belong to each category. Blue and yellow bar show GO terms of biological process and cellular component, respectively. (B) Interactions between DA miRNAs and their mature oocyte target genes involved in catabolic processes. 
A

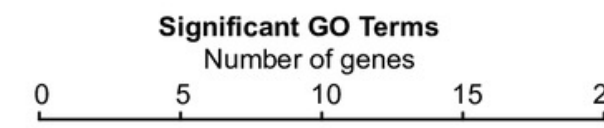

regulation of mRNA processing nucleosome binding nucleosomal DNA binding
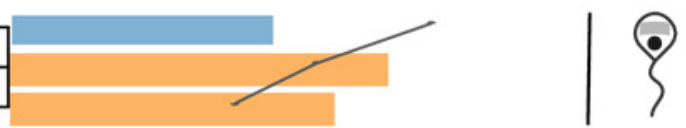

cellular macromolecule catabolic process macromolecule catabolic process cellular protein catabolic process protein catabolic process

ubiquitin-dependent protein catabolic process modification-dependent protein catabolic process proteolysis involved in cellular protein catabolic process modification-dependent macromolecule catabolic process proteasome-mediated ubiquitin-dependent protein catabolic process organonitrogen compound catabolic process proteasomal protein catabolic process macromolecular complex binding translation initiation factor activity actin filament binding
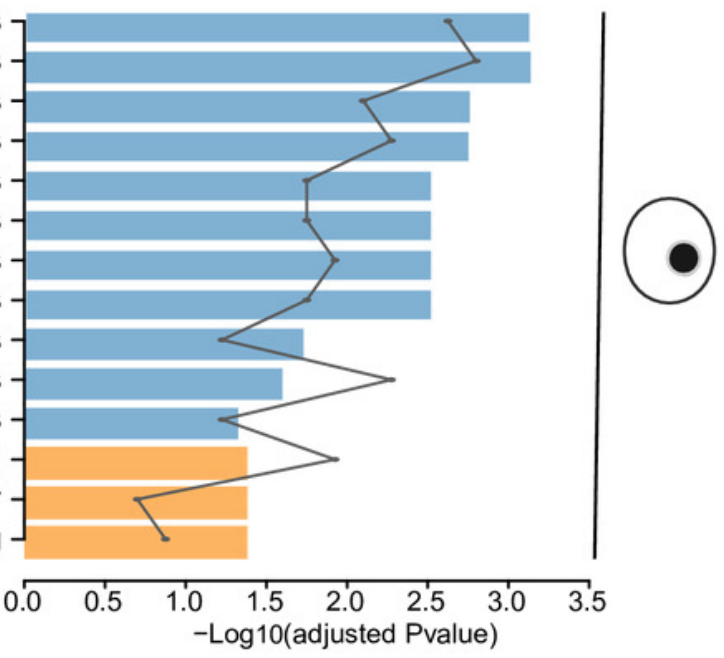

B

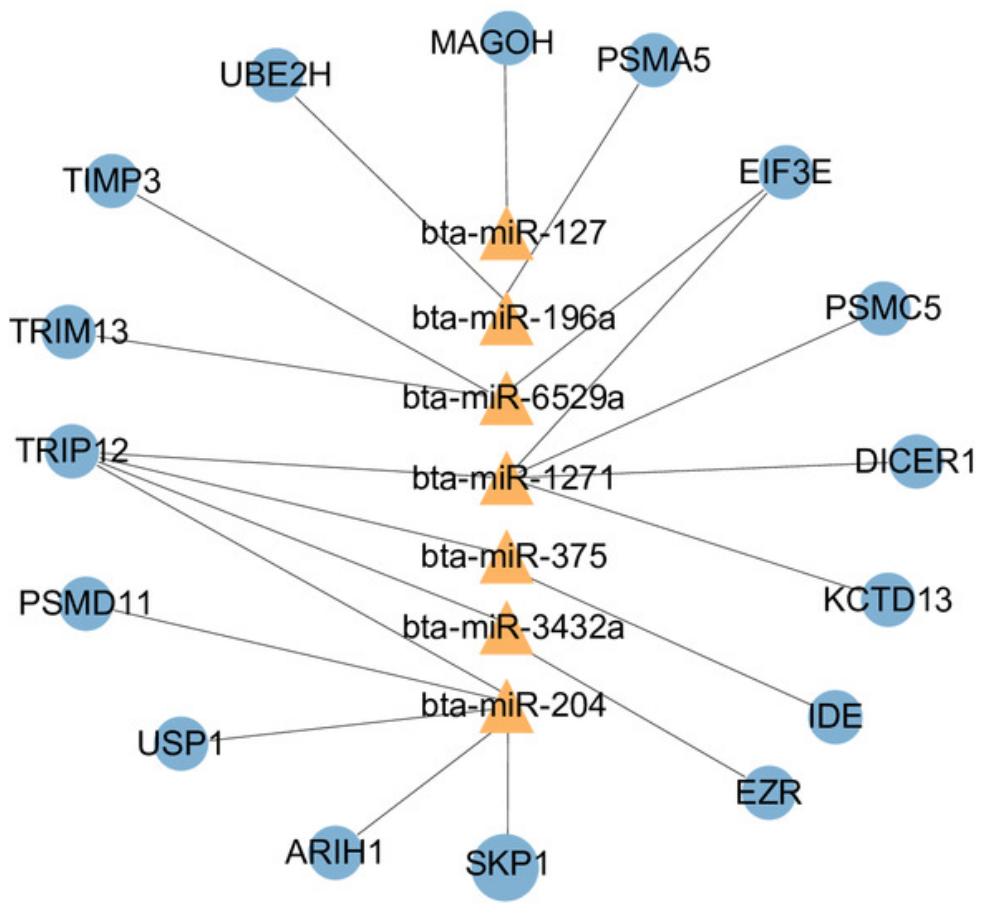

Oocyte gene

DA miRNA 
Figure 3

piRNA clusters and piRNAs of $X$ and $Y$ sperm.

(A) The distribution of piRNA clusters along the chromosomes. The label of $X$ and $Y$ sperm unique clusters are shown by blue red and blue, respectively. (B) Heatmap of clustering to 28 DA piRNAs detected.

A

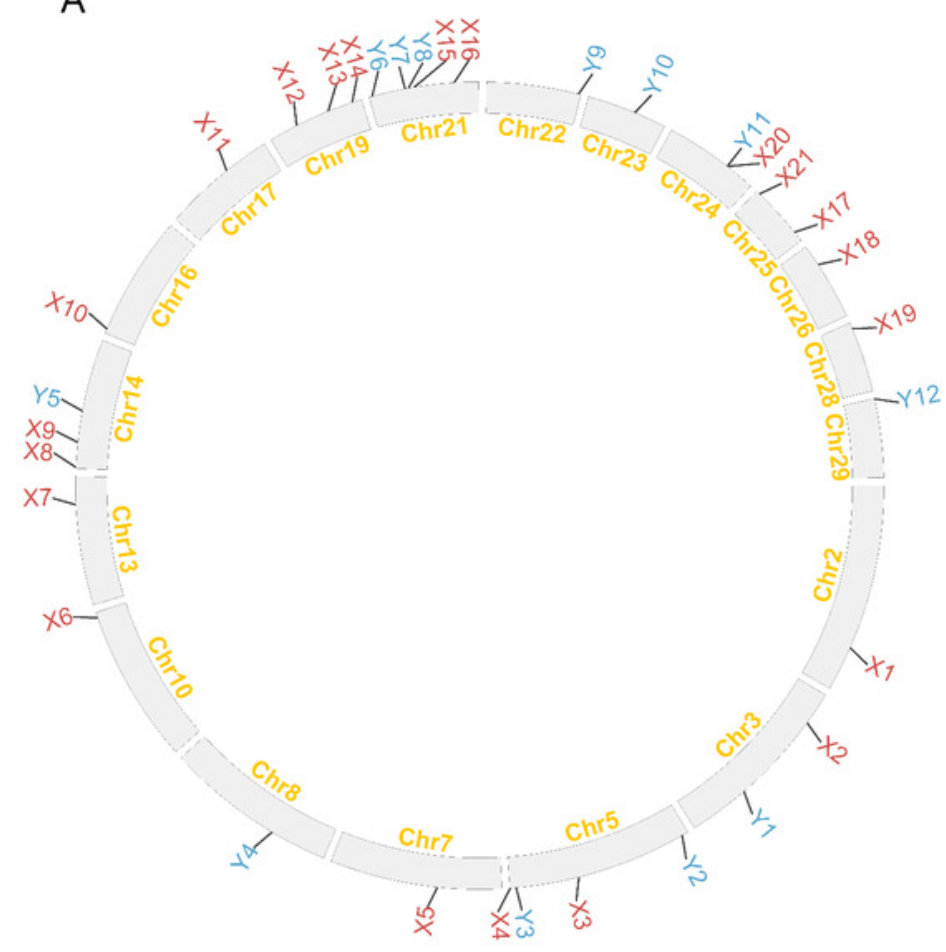

B

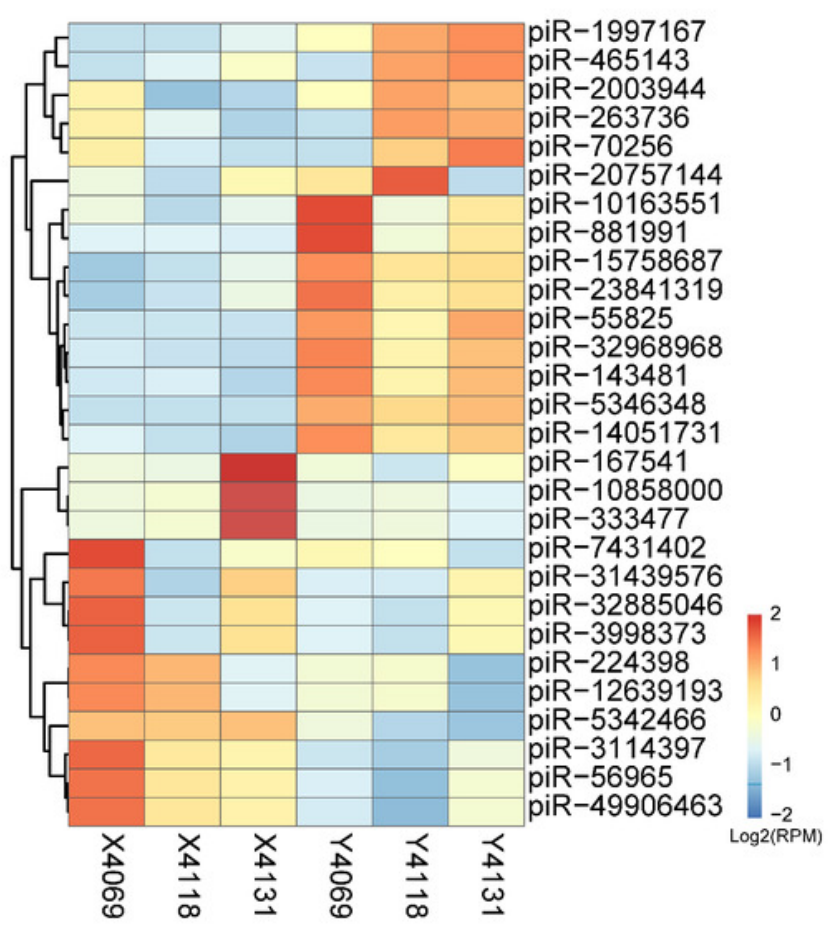


Figure 4

Illustration of the qPCR results for four selected differentially abundant miRNAs. The Xaxis represents four selected miRNAs and Y-axis represents the log2 fold change (X sperm/Y sperm) obtained from qPCR and sequencing. 


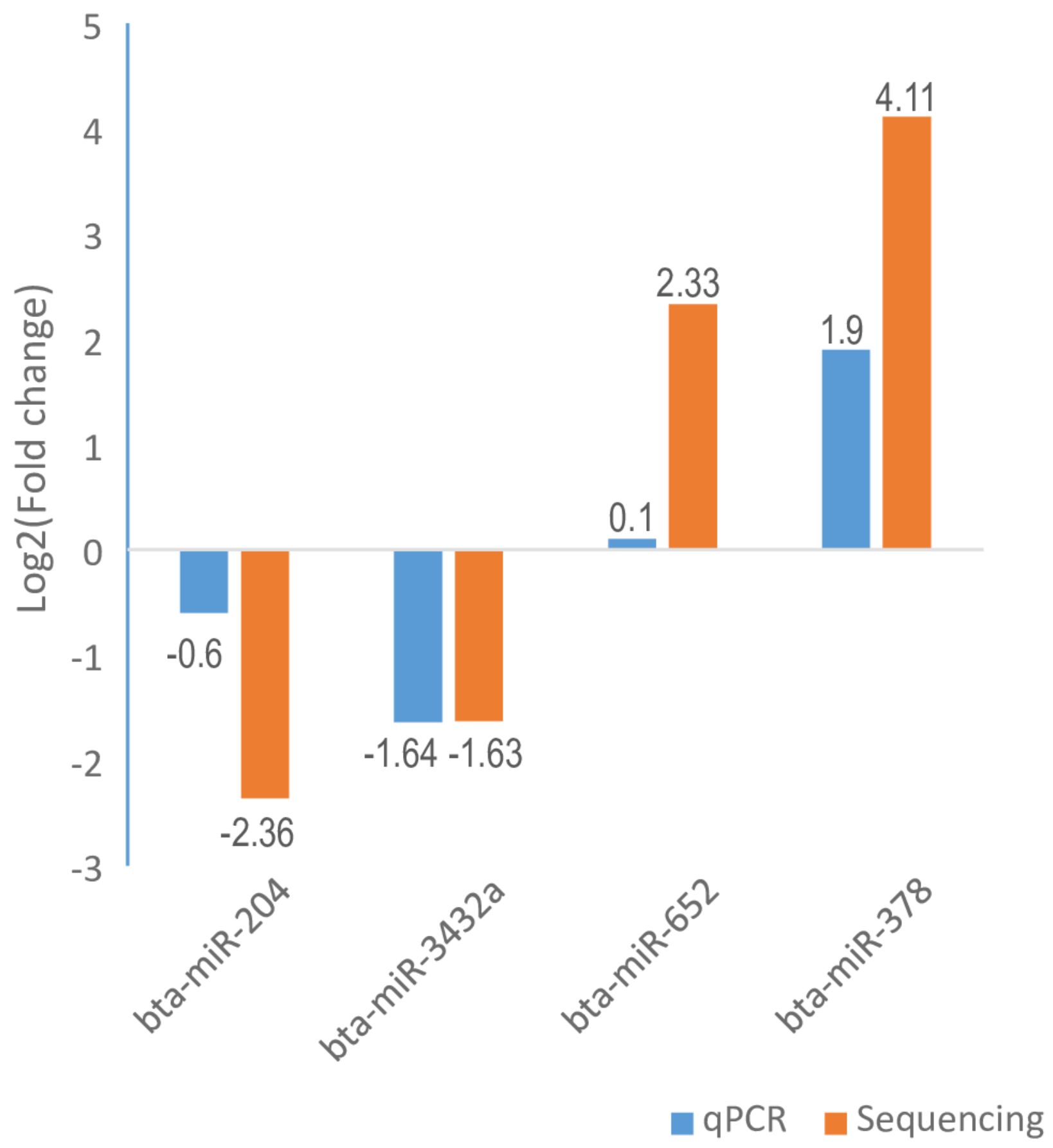




\section{Table $\mathbf{1}$ (on next page)}

Summary of differentially abundant miRNAs between $X$ and $Y$ sperm.

$\log _{2} \mathrm{FC}$ refers to $\log _{2}$ Fold Change, $X$ and $Y$ value refer to the average RPM of miRNA abundance for 3 replications of $X$ and $Y$ sperm. The $\log _{2} F C, P$-value, and RPM values are calculated from the results annotated by Unitas (Table S2). 
1 Table 1. Summary of differentially abundant miRNAs between $\mathrm{X}$ and $\mathrm{Y}$ sperm.

\begin{tabular}{cccccc}
\hline miRNA & $\log _{2} \mathrm{FC}$ & P-value & $\begin{array}{c}\mathrm{X} \\
(\mathrm{RPM})\end{array}$ & $\begin{array}{c}\mathrm{Y} \\
(\mathrm{RPM})\end{array}$ & $\begin{array}{c}\text { Location } \\
\text { (Chr: Start..End) }\end{array}$ \\
\hline bta-miR-204 & -2.36 & 0.0001 & 44289.4 & 159757.7 & $8: 47259591 . .47259612$ \\
bta-miR-1271 & -2.03 & 0.0202 & 30.3 & 72.4 & $7: 39194962 . .39194983$ \\
bta-miR-211 & -1.98 & 0.0076 & 66.9 & 153.3 & $21: 28046473 . .28046493$ \\
bta-miR-375 & -1.76 & 0.0021 & 146.2 & 328.7 & $2: 107667524 . .107667546$ \\
bta-miR-3432a & -1.63 & 0.0164 & 2037.4 & 3111.0 & $21: 55746836 . .55746857$ \\
bta-miR-127 & -1.58 & 0.0262 & 49.0 & 78.3 & $21: 67429798 . .67429819$ \\
bta-miR-6529a & -1.43 & 0.0240 & 1411.7 & 2695.0 & $1: 65453397 . .65453417$ \\
bta-miR-369-5p & -1.34 & 0.0302 & 116.5 & 188.6 & $21: 67603550 . .67603569$ \\
bta-miR-196a & -1.33 & 0.0292 & 129.9 & 205.0 & $19: 38497007.38497028$ \\
bta-miR-15a & 1.47 & 0.0173 & 161.5 & 32.6 & $12: 19596395 . .19596415$ \\
bta-miR-652 & 2.33 & 0.0191 & 293.4 & 27.9 & $30: 62939343 . .62939363$ \\
bta-miR-378 & 4.11 & 0.0000 & 50.7 & 1.6 & $4: 10715305 . .10715326$ \\
\hline
\end{tabular}

$2 \log _{2}$ FC refers to $\log _{2}$ Fold Change, $X$ and $\mathrm{Y}$ value refer to the average RPM of miRNA abundance for 3

3 replications of $\mathrm{X}$ and $\mathrm{Y}$ sperm. The $\log _{2} \mathrm{FC}$, P-value, and RPM values are calculated from the results

4 annotated by Unitas (Table S2).

5 


\section{Table 2 (on next page)}

Comparison of differentially abundant (DA) and highly conserved (Non-DA) miRNAs identified in our study with other studies.

'+' and '-' refer to the miRNAs presented and absented in the datasets of DE miRNAs, non-DE miRNAs,Bull ( Sellem et al.,2020) ,Bull² (Capra et al., 2017), Bull (top 20) (Capra et al., 2017), Human(Pantano et al., 2015), Boar (Chen et al., 2017), and Mouse (Nixon et al., 2015). 
1 Table 2. Comparison of differentially abundant (DA) and highly conserved (Non-DA) miRNAs 2 identified in our study with other studies.

\begin{tabular}{|c|c|c|c|c|c|c|c|c|c|c|}
\hline \multirow{2}{*}{$\begin{array}{c}\text { miRNA } \\
\text { (Bos Taurus) }\end{array}$} & \multicolumn{2}{|c|}{ Reads per million } & \multirow[b]{2}{*}{$\mathrm{DE}$} & \multirow{2}{*}{$\begin{array}{l}\text { No } \\
n- \\
\text { DE }\end{array}$} & \multirow[b]{2}{*}{$\begin{array}{c}\text { Bull }^{1} \\
\text { (Top 20) }\end{array}$} & \multirow[b]{2}{*}{$\begin{array}{c}\text { Bull }^{2} \\
\text { (Top 20) }\end{array}$} & \multicolumn{4}{|c|}{ Previously identified } \\
\hline & $\mathrm{X}$ & $\mathrm{Y}$ & & & & & $\begin{array}{l}\text { Bull }^{2} \\
\text { (All) }\end{array}$ & Human & Boar & Mouse \\
\hline miR-100 & 128833 & 99447 & - & + & + & + & + & + & + & + \\
\hline let $-7 a-5 p$ & 15372 & 13679 & - & + & + & - & - & + & - & + \\
\hline $\operatorname{miR}-22-3 p$ & 18459 & 9877 & - & + & - & + & + & + & + & + \\
\hline miR-151-5p & 16214 & 11325 & - & + & - & + & + & - & + & + \\
\hline $\operatorname{miR}-21-5 p$ & 15621 & 5607 & - & + & + & + & + & + & - & + \\
\hline $\operatorname{miR}-449 a$ & 8210 & 9565 & - & + & - & - & - & + & - & + \\
\hline $\operatorname{miR}-99 a-5 p$ & 9363 & 6399 & - & + & + & - & - & + & - & + \\
\hline miR-16b & 9903 & 3619 & - & + & - & + & + & - & - & - \\
\hline miR-7 & 4595 & 6283 & - & + & + & - & - & - & + & - \\
\hline $\operatorname{miR}-27 a-3 p$ & 7530 & 2925 & - & + & - & + & + & + & - & + \\
\hline miR-127 & 49 & 78 & + & - & - & - & - & + & + & + \\
\hline $\operatorname{miR}-1271$ & 30 & 72 & + & - & - & - & - & - & - & - \\
\hline miR-15a & 162 & 33 & + & - & - & - & - & + & + & + \\
\hline miR-196a & 130 & 205 & + & - & - & - & - & + & + & + \\
\hline miR-204 & 44384 & 159903 & + & - & + & + & + & + & + & + \\
\hline miR-211 & 67 & 153 & + & - & + & - & - & - & - & - \\
\hline miR-3432a & 2042 & 3114 & + & - & - & - & - & - & - & - \\
\hline miR-369-5p & 117 & 189 & + & - & - & - & - & - & - & - \\
\hline miR-375 & 147 & 329 & + & - & + & + & + & + & - & + \\
\hline miR-378 & 51 & 2 & + & - & - & - & - & - & - & + \\
\hline miR-652 & 295 & 28 & + & - & - & - & - & - & - & + \\
\hline $\operatorname{miR}-6529 a$ & 1415 & 2697 & + & - & - & - & - & - & - & - \\
\hline
\end{tabular}

3 '+' and '-' refer to the miRNAs presented and absented in the datasets of DE miRNAs, non-DE miRNAs, Bull ${ }^{1}$

4 (Sellem et al.,2020) ,Bull ${ }^{2}$ (Capra et al., 2017), Bull ${ }^{2}$ (top 20) (Capra et al., 2017), Human(Pantano et al., 5 2015), Boar (Chen et al., 2017), and Mouse (Nixon et al., 2015).

6 